\title{
Evidence from community level inputs to improve quality of care for maternal and newborn health: interventions and findings
}

\author{
Zohra S Lassi ${ }^{1}$, Jai K Das ${ }^{1}$, Rehana A Salam¹, Zulfiqar A Bhutta ${ }^{1,2^{*}}$
}

\begin{abstract}
Annually around 40 million mothers give birth at home without any trained health worker. Consequently, most of the maternal and neonatal mortalities occur at the community level due to lack of good quality care during labour and birth. Interventions delivered at the community level have not only been advocated to improve access and coverage of essential interventions but also to reduce the existing disparities and reaching the hard to reach. In this paper, we have reviewed the effectiveness of care delivered through community level inputs for improving maternal and newborn health outcomes. We considered all available systematic reviews published before May 2013 on the pre-defined community level interventions and report findings from 43 systematic reviews. Findings suggest that home visitation significantly improved antenatal care, tetanus immunization coverage, referral and early initiation of breast feeding with reductions in antenatal hospital admission, cesarean-section rates birth, maternal morbidity, neonatal mortality and perinatal mortality. Task shifting to midwives and community health workers has shown to significantly improve immunization uptake and breast feeding initiation with reductions in antenatal hospitalization, episiotomy, instrumental delivery and hospital stay. Training of traditional birth attendants as a part of community based intervention package has significant impact on referrals, early breast feeding, maternal morbidity, neonatal mortality, and perinatal mortality. Formation of community based support groups decreased maternal morbidity, neonatal mortality, perinatal mortality with improved referrals and early breast feeding rates. At community level, home visitation, community mobilization and training of community health workers and traditional birth attendants have the maximum potential to improve a range of maternal and newborn health outcomes. There is lack of data to establish effectiveness of outreach services, mass media campaigns and community education as standalone interventions. Future efforts should be concerted on increasing the availability and training of the community based skilled health workers especially in resource limited settings where the highest burden exists with limited resources to mobilize.
\end{abstract}

\section{Background}

Annually around 40 million mothers give birth at home without any trained health worker [1]. Consequently, most of the maternal, perinatal and neonatal morbidities and mortalities occur at the community level due to lack of good quality care during labour and birth. The poorest and fragile countries have the highest neonatal mortality rates and preventable deaths depicting the existing inequities $[1,2]$. The causes are multi-factorial, ranging from socio-economic aspects of poverty; poor

\footnotetext{
* Correspondence: zulfiqar.bhutta@aku.edu

'Division of Women \& Child Health, Aga Khan University, Karachi, Pakistan
} Full list of author information is available at the end of the article health status of women; lack of autonomy and decision making authority; and illiteracy to health system related factors like poor antenatal and obstetric care; absence of trained birth attendant; inadequate referral system; lack of transportation facilities; and poor linkages between health centers and communities [1,3]. This burden could be averted by achieving universal coverage in skilled birth attendance and providing good quality care for all births. However due to paucity of trained human resource professionals in first-level health services and the reduced awareness of and accessibility to services for the deprived and marginalized populations, these are not accessible to the ones in need $[1,4]$. 
Community based delivery is now widely recognized as an important strategy to deliver key maternal and child survival interventions [5-10]. It has been instrumental in scaling up coverage of certain interventions, such as immunization, oral rehydration therapy for diarrhea, tuberculosis treatment and family planning initiatives. Interventions delivered at the community level have not only been advocated to improve access and coverage of essential interventions but also to reduce the existing disparities and reaching the hard to reach. Inputs at the community level involve programs based on training and consequent task shifting from healthcare personnel to mid-level health care personnel or lay individuals for local implementation at home, village or any defined community group. They focus on resources such as local knowledge, volunteers' time, community confidence and trust as channels for delivery. Community platforms can be used to deliver a full spectrum of promotive, preventive, and curative interventions including provision of basic antenatal (ANC), natal and postnatal care (PNC); preventive essential newborn care; breastfeeding counseling; management and referral of sick newborns; skills development in behavior change communication; and community mobilization strategies to promote birth and newborn care preparedness. These programs do not substitute for a formal health system, but provide a channel to reach far flung areas with knowledge, commodities and skills, thus attempting to reduce existing inequities in healthcare access and utilization. In this paper, we have reviewed the effectiveness of care delivered through community level inputs for improving maternal newborn health $(\mathrm{MNH})$ outcomes. For this review, we have broadly categorized these interventions into four categories: outreach services (including home visitation and referrals); task shifting; training; and formation of support groups for community mobilization.

\section{Community level characteristics \\ Outreach services and home visitation}

Home-based strategies to support optimal maternal and neonatal care practices have emerged in the last decade to complement facility-based care and promote universal access to and utilization of health services with a specific focus on low- and middle- income countries (LMICs). These services mainly include ANC, skilled birth attendance and early PNC. Such programs involve standardized or individualized interventions for additional support provided during home visits, regular ANC visits, and/or by telephone throughout pregnancy by multidisciplinary teams of health professionals and trained lay workers. The major benefit of these programs is that the service is brought to the remote population in their own homes and allow care providers to deliver a more tailored health care service.

\section{Task shifting}

Task shifting for human resource management involves substituting specialized personnel with healthcare workers that are lesser trained but can perform some aspects of their tasks. A range of both skilled and semi-skilled health workers can play a major role in $\mathrm{MNH}$ service delivery. Community health workers $(\mathrm{CHW})$ and traditional birth attendants (TBA) are considered semi-skilled workers, while mid-level health workers (MLHW) such as nurses, midwives, associate clinicians, medical assistants and nurse auxiliaries are skilled workers certified for their work. Health service delivery through these skilled and semi-skilled healthcare workers has been practiced in both high-income countries (HIC) and LMIC all over the world for the past several decades. Evidence suggests that they can contribute significantly in improving health of the populations. More recently, due to the growing human resource crisis especially in LMICs, task shifting has reemerged for extending services to hard-to-reach groups by substituting health professionals for a range of tasks [11-14]. Countries in south Asia and Africa have made a particular effort in recent years to reduce maternal and neonatal mortality and morbidity through deploying CHW $[15,16]$. The role of midwives and TBA in delivering $\mathrm{MNH}$ services has also received growing attention in the last few years, and a number of publications have described their role and documented the effects of such programs $[17,18]$. However, less attention has been given to assess the effectiveness of MLHW in delivering and improving health care delivery [19].

\section{Human resource training}

Globally there is a growing shortage of 7.2 million healthcare workers and around $90 \%$ of all maternal deaths and $80 \%$ of still births occur in countries that lack trained healthcare workforce [20]. Although skillsmix imbalances persist, advanced practitioners, midwives, nurses and auxiliaries are still insufficiently used in many settings. Many LMIC are increasingly facing difficulties in producing, recruiting and retaining health professionals as they tend to migrate to wealthier countries due to low salaries, poor working conditions, lack of supervision, low morale and motivation and lack of infrastructure $[9,21,22]$. To overcome the failure of providing birthing women with skilled attendance, poor countries are now investing on training MLHW to at least provide them with some sort of assistance instead of none at all. In countries like Malawi, Bangladesh, Pakistan and Guatemala, training and close supervision of TBA have been evaluated to improve MNH outcomes and have shown some potential in reducing harmful practices during delivery and childbirth and improving $\mathrm{MNH}$ outcomes $[23,24]$. These training courses include short and structured approach to equip lay workers with lifesaving tools 
like Newborn Life Support (NLS), Neonatal Resuscitation Program (NRP) and Essential Newborn Care (ENC) but might vary in origin, scope and target audience. Besides additional training of outreach workers, human resource training also includes conferences, lectures, workshops, group education, seminars and symposia.

\section{Community mobilization and support groups}

Soon after the Alma-Ata Declaration, it was recognized that community participation was important for the provision of local health services and for delivering interventions at the community level. Since then, it has been advocated to build its links with improving $\mathrm{MNH}$ [25]. Community support groups and women's groups are now increasingly becoming a core component of community service package comprising of community representatives for health promotion. The objective is to enable the community to provide support to pregnant women and families throughout pregnancy and delivery. Communities are encouraged to identify key barriers to care and select the most appropriate interventions for their situation. Community mobilization also helps educate about available services, identification of danger signs during pregnancy, and the importance of seeking care from skilled healthcare worker during obstetric and newborn emergencies. A range of promotive messages, quality care and scale up coverage for $\mathrm{MNH}$ can be delivered through community workers and women's groups [26].

In this review, we aim to systematically review and summarize the available evidence from relevant systematic reviews on the impact of the outlined community level inputs Figure 1). to improve the quality of care for women and newborns.

\section{Methods}

We considered all available systematic reviews published before May 2013 on the pre-defined community level interventions as outlined in our conceptual framework [27]. A separate search strategy was developed for each component using broad keywords, medical subject heading $(\mathrm{MeSH})$, and free text terms: [(Community OR home OR "home visit"” OR outreach OR "task shift" OR

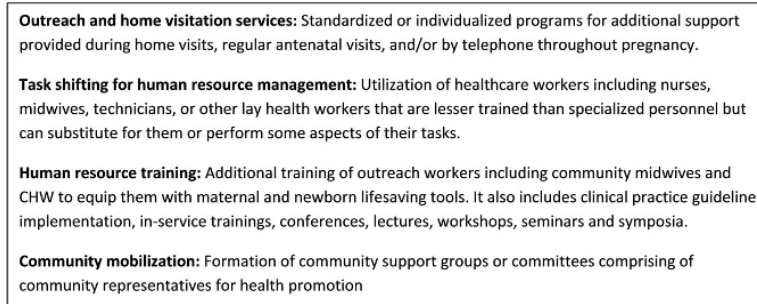

Figure 1 Components of community level interventions "human resource" OR "in-service" OR training OR mobilization OR "support group" "OR "women's group" OR "health worker" ${ }^{*}$ OR "community health aides" OR "primary health care" OR "community health worker"” OR "lay health worker" OR "mid-level health worker*" OR "community based interventions") AND (health OR maternal OR mother OR child OR newborn OR neonat*)]

Our priority was to select existing systematic reviews of randomized or non-randomized controlled trials, which fully or partly address the a priori defined community delivered interventions for improving quality of care for $\mathrm{MNH}$. We excluded reviews on home visits for prevention or screening for child abuse, maltreatment and childhood injury prevention as these were not included in the scope of our review. Search was conducted in the Cochrane library and PubMed and reviews that met the inclusion criteria were selected and data was abstracted by two authors on a standardized abstraction sheet. Quality assessment of the included reviews was done using Assessment of Multiple Systematic Reviews (AMSTAR) criteria [28] as detailed in paper 1 [27]. Any disagreement between the primary abstractors was resolved by the third author. For the pre-identified interventions, which did not specifically report $\mathrm{MNH}$ outcomes, we have reported the impacts on other health outcomes reported by the review authors. Estimates are reported as relative risks (RR), odds ratios (OR), risk differences (RD) or mean differences (MD) with 95\% confidence intervals (CI) where available. For detailed methodology please refer to paper 1 of the series [27].

\section{Findings}

Our search yielded 310 potentially relevant review titles. Further screening of abstracts and full texts resulted in the inclusion of 43 eligible reviews: 17 for outreach services (home visitation and referrals), 6 for task shifting, 18 for human resource training and 2 for community mobilization (Figure 2). The overall quality of the reviews ranged from 3 to 11 with a median of 9 on the AMSTAR criteria.

\section{Outreach Services}

We included 16 [29-44] reviews and 1 [45] overview of reviews pertaining to outreach and home visitation services with the median quality score of 7 on AMSTAR criteria. All reviews except one [33] reported MNH specific outcomes. The most commonly reported outcomes included maternal, newborn morbidity and mortality, immunization rates, breast feeding, referral, ANC and PNC utilization. Meta-analysis was conducted in six of the reviews. Reviews evaluating the impact of structured nurse- or midwife-based home visitation programs were from HIC while those focusing on service delivery through CHW were from LMIC. Table 1 summarizes the characteristics of the included reviews. 


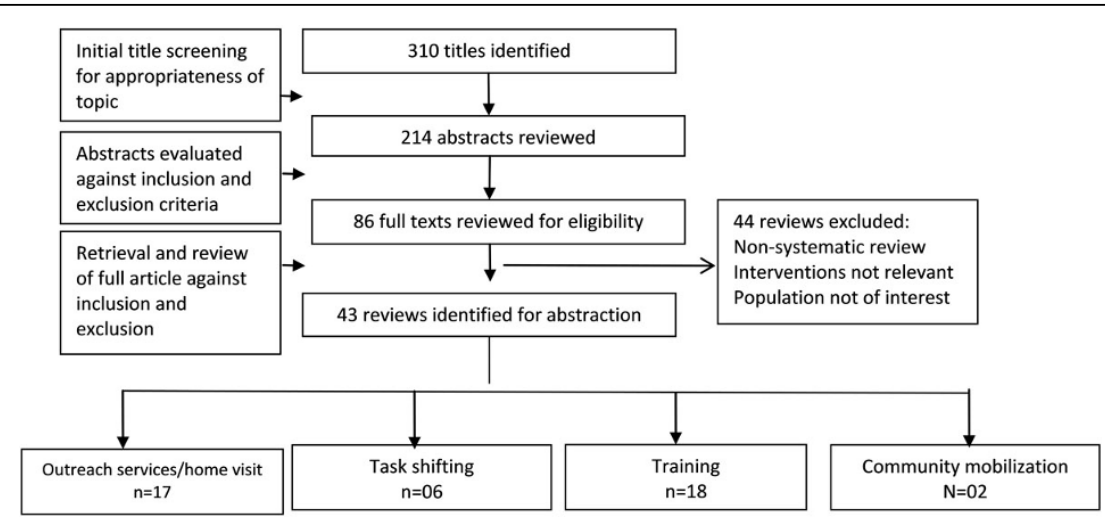

Figure 2 Search flow diagram

Table 1 Characteristics of the included reviews for outreach services, home visitation and referrals

\begin{tabular}{|c|c|c|c|c|c|c|c|}
\hline \multirow{2}{*}{$\begin{array}{c}\text { Reviews } \\
(n=16)\end{array}$} & \multirow{2}{*}{$\begin{array}{l}\text { Description of included } \\
\text { interventions }\end{array}$} & \multirow{2}{*}{$\begin{array}{c}\text { Type of } \\
\text { studies } \\
\text { included (no) }\end{array}$} & \multirow{2}{*}{$\begin{array}{l}\text { Targeted health } \\
\text { care providers }\end{array}$} & \multicolumn{2}{|c|}{ Outcome reported } & \multirow{2}{*}{$\begin{array}{l}\text { Pooled } \\
\text { data } \\
(\mathrm{Y} / \mathrm{N})\end{array}$} & \multirow[t]{2}{*}{ Results } \\
\hline & & & & $\begin{array}{l}\text { Other } \\
\text { outcomes }\end{array}$ & $\begin{array}{l}\text { MNCH specific } \\
\text { outcomes }\end{array}$ & & \\
\hline \multirow[t]{2}{*}{$\begin{array}{c}\text { Blondel } 1995 \\
{[29]}\end{array}$} & $\begin{array}{l}\text { Two different types of } \\
\text { home visits during } \\
\text { pregnancy: (1) those } \\
\text { offering social support to } \\
\text { high-risk women; and (2) } \\
\text { those providing medical } \\
\text { care to women with } \\
\text { complications. }\end{array}$ & RCT's: 08 & $\begin{array}{l}\text { Nurses, family } \\
\text { workers, } \\
\text { midwives in HIC }\end{array}$ & & Preterm delivery & Yes & $1.0(0.8-1.1)$ \\
\hline & & & & & $\begin{array}{l}\text { Hospital admission } \\
\text { with complications }\end{array}$ & & $0.9(0.7-1.2)$ \\
\hline \multirow[t]{5}{*}{$\begin{array}{c}\text { Bull 2004 } \\
\text { (Overview) } \\
{[45]}\end{array}$} & $\begin{array}{l}\text { Home visiting is not a } \\
\text { single or uniform } \\
\text { intervention - it is a } \\
\text { mechanism for the delivery } \\
\text { of a variety of interventions } \\
\text { directed at different } \\
\text { outcomes. They may } \\
\text { provide parent training/ } \\
\text { education, pyscho-social } \\
\text { support to parents, infant } \\
\text { stimulation, and infant and } \\
\text { maternal health surveillance }\end{array}$ & Reviews: 09 & $\begin{array}{l}\text { Nurses, midwives } \\
\text { or lay people } \\
\text { within different } \\
\text { professional } \\
\text { bases in HIC }\end{array}$ & & $\begin{array}{l}\text { Pregnancy } \\
\text { outcome }\end{array}$ & No & No impact \\
\hline & & & & & Immunization rate & & No impact \\
\hline & & & & & Hospital admission & & No impact \\
\hline & & & & & Child injury & & Positive impact \\
\hline & & & & & $\begin{array}{l}\text { Post natal } \\
\text { depression }\end{array}$ & & Positive impact \\
\hline $\begin{array}{c}\text { Ciliska } 2001 \\
{[30]}\end{array}$ & $\begin{array}{l}\text { Public health nursing } \\
\text { interventions when carried } \\
\text { out by the strategy of } \\
\text { home visiting of clients in } \\
\text { the pre- and postnatal } \\
\text { period }\end{array}$ & $\begin{array}{l}20 \text { studies } \\
\text { RCT's: } 8 \\
\text { CCT: } 3 \text { analytic } \\
\text { cohort: } 1\end{array}$ & $\begin{array}{l}\text { Nurses or } \\
\text { midwives in HIC }\end{array}$ & & $\begin{array}{l}\text { Children's mental } \\
\text { development, } \\
\text { mental health and } \\
\text { physical growth, } \\
\text { mother's } \\
\text { depression, } \\
\text { maternal } \\
\text { employment, } \\
\text { education, nutrition } \\
\text { and other health } \\
\text { habits, and } \\
\text { government cost } \\
\text { saving. }\end{array}$ & No & $\begin{array}{l}\text { No negative impacts } \\
\text { reported in } 12 \text { strong } \\
\text { articles }\end{array}$ \\
\hline
\end{tabular}




\begin{tabular}{|c|c|c|c|c|c|c|c|}
\hline & & & & & & & $\begin{array}{l}\text { Children mental and } \\
\text { physical health } \\
\text { improved } \\
\text { No impacts on LBW, } \\
\text { gestational age and } \\
\text { neonatal morbidity } \\
\text { and mortality }\end{array}$ \\
\hline \multirow[t]{9}{*}{$\begin{array}{c}\text { Elkan } 2000 \\
{[31]}\end{array}$} & $\begin{array}{c}\text { Home visiting program } \\
\text { with at least one postnatal } \\
\text { visit }\end{array}$ & $\begin{array}{c}102 \text { papers } \\
\text { with } 86 \text { home } \\
\text { visiting } \\
\text { programs }\end{array}$ & $\begin{array}{c}\text { Nurses or } \\
\text { midwives in HIC }\end{array}$ & & $\begin{array}{c}\text { Mental } \\
\text { development score }\end{array}$ & Yes & $0.17(0.06-0.28)$ \\
\hline & & & & & $\begin{array}{c}\text { Motor } \\
\text { development score }\end{array}$ & & $0.17(-0.03-0.38)$ \\
\hline & & & & & IQ & & $0.32(0.146-0.48)$ \\
\hline & & & & & Weight & & $0.04(-0.17-2.46)$ \\
\hline & & & & & Height & & $0.04(-0.17-2.5)$ \\
\hline & & & & & Immunization rate & & $1.40(1.16-1.68)$ \\
\hline & & & & & Use of acute care & & $0.73(0.55-0.98)$ \\
\hline & & & & & Hospital stay & & $1.63(1.18-2.24)$ \\
\hline & & & & & ER & & $0.77(0.58-1.03)$ \\
\hline \multirow[t]{13}{*}{$\underset{[32]}{\operatorname{Gogia} 2010}$} & $\begin{array}{l}\text { Implementation by } \\
\text { community health workers } \\
\text { of safe delivery practices at } \\
\text { home and proper care of } \\
\text { the neonate immediately } \\
\text { after birth, such as keeping } \\
\text { the baby warm, providing } \\
\text { neonatal resuscitation (if } \\
\text { required) and initiating } \\
\text { breastfeeding early. }\end{array}$ & RCT: 05 & CHW in LIC & & ANC visit $>1$ & Yes & $1.33(1.20-1.47)$ \\
\hline & & & & & $\begin{array}{c}\text { Tetanus Toxoid (2 } \\
\text { doses) }\end{array}$ & & $1.11(1.04-1.18)$ \\
\hline & & & & & Skilled care at birth & & $1.54(0.81-2.93)$ \\
\hline & & & & & $\begin{array}{l}\text { Breastfeeding } \\
\text { within } 1 \text { hour }\end{array}$ & & $3.35(1.31-8.59)$ \\
\hline & & & & & Clean cord care & & $1.70(1.39-2.07)$ \\
\hline & & & & & Delayed bathing & & $4.63(2.29-9.37)$ \\
\hline & & & & & Neonatal mortality & & $0.62(0.44-0.87)$ \\
\hline & & & & & Infant mortality & & $0.41(0.30-0.57)$ \\
\hline & & & & & $\begin{array}{l}\text { Neonatal cause- } \\
\text { specific mortality } \\
\text { due to: }\end{array}$ & & \\
\hline & & & & & Sepsis & & $89.8 \%(78.6-101.0)$ \\
\hline & & & & & Asphyxia & & $53.3 \%(23.8-82.8)$ \\
\hline & & & & & Prematurity & & $38 \%(4.3-71.6)$ \\
\hline & & & & & Hypothermia & & $\begin{array}{c}\text { 100\% (one-sided 95\% } \\
\text { Cl not stated) }\end{array}$ \\
\hline \multirow[t]{2}{*}{$\begin{array}{c}\text { Gruen } 2003 \\
{[33]}\end{array}$} & $\begin{array}{l}\text { Specialist outreach clinics: } \\
\text { defined as planned and } \\
\text { regular visits by specialist- } \\
\text { trained medical } \\
\text { practitioners from a usual } \\
\text { practice location (hospital } \\
\text { or specialist center) to } \\
\text { primary care or rural } \\
\text { hospital settings. }\end{array}$ & $\begin{array}{l}\text { RCT: } 05 \\
\text { CBA: } 02 \\
\text { ITS: } 02\end{array}$ & $\begin{array}{c}\text { Primary } \\
\text { healthcare } \\
\text { practitioners and } \\
\text { specialists }\end{array}$ & $\begin{array}{l}\text { Adherence } \\
\text { to } \\
\text { treatment }\end{array}$ & & Yes & $0.63(0.52-0.77)$ \\
\hline & & & & $\begin{array}{l}\text { Patient and } \\
\text { provider } \\
\text { satisfaction }\end{array}$ & & & $0.43(0.29-0.62)$ \\
\hline
\end{tabular}


Table 1 Characteristics of the included reviews for outreach services, home visitation and referrals (Continued)

\begin{tabular}{|c|c|c|c|c|c|c|c|}
\hline & & & & $\begin{array}{l}\text { Use of } \\
\text { service }\end{array}$ & & & $0.14(0.05-0.32)$ \\
\hline \multirow[t]{9}{*}{$\begin{array}{l}\text { Hodnett } \\
2000[34]\end{array}$} & $\begin{array}{l}\text { Standardized or } \\
\text { individualized programs of } \\
\text { additional social support } \\
\text { provided in either home } \\
\text { visits, during regular } \\
\text { antenatal clinic visits, and/ } \\
\text { or by telephone on several } \\
\text { occasions during } \\
\text { pregnancy. }\end{array}$ & RCT: 17 & $\begin{array}{l}\text { Multidisciplinary } \\
\text { teams of health } \\
\text { professionals } \\
\text { specially trained } \\
\text { lay workers, or } \\
\text { combination of } \\
\text { lay and } \\
\text { professional } \\
\text { workers. }\end{array}$ & & Caesarean birth & & $0.87(0.78-0.97)$ \\
\hline & & & & & $\begin{array}{c}\text { Gestational age less } \\
\text { than } 37 \text { weeks at } \\
\text { birth }\end{array}$ & & $0.92(0.83-1.01)$ \\
\hline & & & & & $\begin{array}{l}\text { Birth weight less } \\
\text { than } 2500 \mathrm{gm}\end{array}$ & & $0.92(0.83-1.03)$ \\
\hline & & & & & $\begin{array}{l}\text { Stillbirth/neonatal } \\
\text { death }\end{array}$ & & $0.96(0.74-1.26)$ \\
\hline & & & & & $\begin{array}{c}\text { Antenatal hospital } \\
\text { admission }\end{array}$ & & $0.79(0.68-0.92)$ \\
\hline & & & & & $\begin{array}{l}\text { Postnatal re- } \\
\text { hospitalization }\end{array}$ & & $1.60(0.80-3.21)$ \\
\hline & & & & & $\begin{array}{l}\text { Antenatal } \\
\text { depression }\end{array}$ & & $0.77(0.50,1.19)$ \\
\hline & & & & & $\begin{array}{l}\text { Postnatal } \\
\text { depression }\end{array}$ & & $0.85(0.69-1.05)$ \\
\hline & & & & & $\begin{array}{l}\text { Less than highly } \\
\text { satisfied with } \\
\text { antenatal care }\end{array}$ & & $1.13(0.76,1.67)$ \\
\hline \multirow[t]{2}{*}{$\begin{array}{c}\text { Hussein } 2012 \\
{[35]}\end{array}$} & $\begin{array}{l}\text { Interventions included } \\
\text { aimed to overcome delays } \\
\text { in reaching the appropriate } \\
\text { facility, which improved } \\
\text { emergency referrals } \\
\text { antenatally, during labour, } \\
\text { or up to } 42 \mathrm{~d} \text { after delivery. }\end{array}$ & $\begin{array}{c}\text { Total: } 19 \\
\text { RCT: 04, } \\
\text { controlled } \\
\text { before after: } \\
\text { 05,Cohort: } 05\end{array}$ & $\begin{array}{l}\text { Community } \\
\text { groups and TBA }\end{array}$ & & Neonatal mortality & No & $0.48(0.34-0.68)$ \\
\hline & & & & & Stillbirths & & $0.56(0.32-0.96)$ \\
\hline \multirow[t]{2}{*}{$\begin{array}{c}\text { Hussein } 2012 \\
{[36]}\end{array}$} & $\begin{array}{l}\text { Refer pregnant and post- } \\
\text { partum women suffering } \\
\text { from an emergency } \\
\text { obstetric complication or } \\
\text { from home to basic-level } \\
\text { health facilities (health } \\
\text { centres) and from health } \\
\text { centre to hospital (but not } \\
\text { referral between hospitals) } \\
\text { in LMIC }\end{array}$ & $\begin{array}{c}\text { Total: } 19 \\
\text { RCT: 04, } \\
\text { controlled } \\
\text { before after: } \\
\text { 05,Cohort: } 05\end{array}$ & $\begin{array}{l}\text { Community } \\
\text { groups and TBA }\end{array}$ & & Neonatal mortality & No & $0.48(0.34-0.68)$ \\
\hline & & & & & Stillbirths & & $0.56(0.32-0.96)$ \\
\hline \multirow[t]{3}{*}{$\begin{array}{c}\text { Issel } 2011 \\
{[37]}\end{array}$} & $\begin{array}{l}\text { Prenatal home visiting was } \\
\text { defined as a nonmedical } \\
\text { program or service focused } \\
\text { on facilitating utilization of } \\
\text { health or social services, } \\
\text { provided in the home to } \\
\text { pregnant women who } \\
\text { were at high medical or } \\
\text { social risk for adverse birth } \\
\text { outcomes. }\end{array}$ & $\begin{array}{l}\text { Total : } 28 \\
\text { RCT: } 14, \\
\text { descriptive: } 2, \\
\text { retro cohort: } \\
\text { 07, prospect } \\
\text { cohort: } 02 \text {, } \\
\text { matched CC: } \\
\text { 01, ecological : } \\
\text { 01, static } \\
\text { group: } 01\end{array}$ & $\begin{array}{l}\text { Home visiting } \\
\text { personnel not } \\
\text { defined }\end{array}$ & & PNC utilization & No & $\begin{array}{l}5 / 5 \text { studies found } \\
\text { significant } \\
\text { improvement }\end{array}$ \\
\hline & & & & & Gestational age & & $\begin{array}{c}\text { 5/24 found a } \\
\text { significant positive } \\
\text { effect }\end{array}$ \\
\hline & & & & & Birth weight & & $\begin{array}{c}\text { 7/17 found a } \\
\text { significant positive } \\
\text { effect }\end{array}$ \\
\hline
\end{tabular}


Table 1 Characteristics of the included reviews for outreach services, home visitation and referrals (Continued)

\begin{tabular}{|c|c|c|c|c|c|c|c|}
\hline \multirow[t]{2}{*}{$\begin{array}{l}\text { Kandrick } \\
2000[38]\end{array}$} & $\begin{array}{c}\text { The home visitation } \\
\text { program had to include at } \\
\text { least one post natal home } \\
\text { visits }\end{array}$ & $\begin{array}{c}11 \text { RCT's }(9 \\
\text { meta-analyzed) }\end{array}$ & $\begin{array}{c}\text { Nurses and } \\
\text { midwives in } \mathrm{HIC}\end{array}$ & & $\begin{array}{l}\text { Immunization } \\
\text { uptake }\end{array}$ & Yes & $\begin{array}{l}1.17 \text { (0.33-4.17) } \\
\text { (Random) }\end{array}$ \\
\hline & & & & & & & $1.67(1.29-2.15)$ (fixed) \\
\hline \multirow[t]{6}{*}{$\begin{array}{c}\text { Lassi } 2010 \\
{[39]}\end{array}$} & $\begin{array}{l}\text { Intervention packages that } \\
\text { included additional training } \\
\text { of outreach workers in } \\
\text { maternal care during } \\
\text { pregnancy, delivery and in } \\
\text { the postpartum period; and } \\
\text { routine newborn care. }\end{array}$ & $\begin{array}{l}18 \text { cluster- } \\
\text { randomized/ } \\
\text { quasi- } \\
\text { randomized } \\
\text { trials }\end{array}$ & $\begin{array}{c}\text { outreach } \\
\text { workers in LMIC }\end{array}$ & & Maternal mortality & Yes & $0.77(0.59-1.02)$ \\
\hline & & & & & Maternal morbidity & & $0.75(0.61-0.92)$ \\
\hline & & & & & Neonatal mortality & & $0.76(0.68-0.84)$ \\
\hline & & & & & Perinatal mortality & & $0.80(0.71-0.91)$ \\
\hline & & & & & Referral & & $1.4(1.19-1.65)$ \\
\hline & & & & & Early breast feeding & & $1.94(1.56-2.42)$ \\
\hline $\begin{array}{l}\text { Lonkhuijzen } \\
\quad 12[44]\end{array}$ & $\begin{array}{l}\text { All types of facilities within } \\
\text { easy reach of a medical } \\
\text { facility that are designated } \\
\text { for the lodging of pregnant } \\
\text { women who await labour, } \\
\text { with the purpose of the } \\
\text { women being assisted by } \\
\text { skilled attendants during } \\
\text { delivery }\end{array}$ & None & Not applicable & $\begin{array}{c}\text { Not } \\
\text { applicable }\end{array}$ & & & \\
\hline $\begin{array}{l}\text { McNaughton } \\
2004[40]\end{array}$ & $\begin{array}{l}\text { Home-visiting interventions } \\
\text { using professional nurses as } \\
\text { home visitors. }\end{array}$ & 13 reports & Nurses in $\mathrm{HIC}$ & & $\begin{array}{l}\text { Maternal newborn } \\
\text { health status }\end{array}$ & No & $\begin{array}{l}\text { Narrative (more than } \\
\text { half of the studies } \\
\text { were able to achieve } \\
\text { their desired results) }\end{array}$ \\
\hline \multirow[t]{4}{*}{$\begin{array}{l}\text { Peacock } \\
2013[43]\end{array}$} & $\begin{array}{l}\text { Effect of paraprofessional } \\
\text { home-visiting programs on } \\
\text { developmental and health } \\
\text { outcomes of young } \\
\text { children from } \\
\text { disadvantaged families. }\end{array}$ & 21 studies & $\begin{array}{l}\text { Paraprofessional } \\
\text { home-visiting } \\
\text { staff }\end{array}$ & & $\begin{array}{l}\text { Child abuse and } \\
\text { neglect }\end{array}$ & No & $\begin{array}{l}3 \text { out of } 6 \text { studies } \\
\text { showed better } \\
\text { outcomes }\end{array}$ \\
\hline & & & & & Physical growth & & $\begin{array}{l}5 \text { out of } 7 \text { studies } \\
\text { showed no } \\
\text { significant } \\
\text { improvement }\end{array}$ \\
\hline & & & & & $\begin{array}{l}\text { Hospitalization, } \\
\text { illness and injuries }\end{array}$ & & $\begin{array}{c}2 \text { out of } 6 \text { studies } \\
\text { showed better health } \\
\text { outcomes }\end{array}$ \\
\hline & & & & & $\begin{array}{l}\text { Up-to date } \\
\text { immunizations }\end{array}$ & & $\begin{array}{c}1 \text { study showed } \\
\text { intervention group } \\
\text { more likely to receive } \\
\text { primary } \\
\text { immunizations }\end{array}$ \\
\hline $\begin{array}{c}\text { Pyone } 2012 \\
{[41]}\end{array}$ & $\begin{array}{l}\text { Distance and transport cost } \\
\text { related interventions }\end{array}$ & 5 studies & Community & & $\begin{array}{l}\text { MMR (associated } \\
\text { with distance) }\end{array}$ & No & $7.4(1.6-132.4)$ \\
\hline \multirow[t]{5}{*}{$\underset{[42]}{\text { Vieira } 2012}$} & $\begin{array}{l}\text { Interventions to increase } \\
\text { birth with skilled health } \\
\text { personnel, in settings } \\
\text { where TBAs were providers } \\
\text { of childbirth care }\end{array}$ & $\begin{array}{c}6 \\
\text { observational } \\
\text { studies }\end{array}$ & $\begin{array}{l}\text { Skilled birth } \\
\text { attendant }\end{array}$ & & $\begin{array}{l}\text { Obstetric mortality } \\
\text { ratio }\end{array}$ & No & $\begin{array}{l}\text { Deploying skilled } \\
\text { health personnel and } \\
\text { addressing financial } \\
\text { barriers for users } \\
\text { increased the use of } \\
\text { skilled health } \\
\text { personnel at birth }\end{array}$ \\
\hline & & & & & $\begin{array}{l}\text { Decrease in } \\
\text { maternal deaths }\end{array}$ & & \\
\hline & & & & & Birth by a physician & & \\
\hline & & & & & Birth by C-Section & & \\
\hline & & & & & $\begin{array}{l}\text { Increase in skilled } \\
\text { birth attendance }\end{array}$ & & \\
\hline
\end{tabular}


Home visits by $\mathrm{CHW}$ to improve neonatal health was associated with improved ANC (RR: 1.33, 95\% CI: 1.201.47), tetanus immunization coverage(RR: $1.11,95 \% \mathrm{CI}$ : 1.04-1.18), breast feeding initiation within 1 hour (RR: 3.35, 95\% CI: 1.31-8.59) and clean cord care (RR: 1.70, 95\% CI: 1.39-2.07) [32]. Community based packages with an emphasis on provision of care through trained $\mathrm{CHW}$ via home visitation significantly improved maternal morbidity (RR: $0.75,95 \%$ CI: 0.61-0.92), neonatal mortality (RR: 0.76 95\% CI: 0.68-0.84), perinatal mortality (RR: 0.80, 95\% CI: 0.71-0.91), referral (RR: 1.4, 95\% CI: 1.19-1.65) and early breast feeding initiation (RR: 1.94, 95\% CI: $1.56-$ 2.42) [39]. A review evaluating the effectiveness of emergency obstetric referral interventions in LMIC showed that community based interventions for generating funds for transport reduced neonatal deaths in India (OR: 0.48, 95\% CI: 0.34-0.68) while maternity waiting home interventions in sub-Saharan Africa reported reductions in stillbirths (OR: 0.56, 95\% CI: 0.32-0.96) [35]. Another review to assess the effects of a maternity waiting facility on maternal and perinatal health did not find any trial for inclusion [44].

Nurse- or midwives- based home visit programs did not report any significant impact on birth outcomes, hospital admission for complications and neonatal morbidity and mortality. However, some positive impacts were reported on immunization rates (RR: 1.67, 95\% CI:
1.29-2.15); child health outcomes including mental and physical health; and injury prevention $[29,30,38,45]$. Home visits with a specific focus on post natal visit was found to be associated with improved immunization rates (RR: 1.40, 95\% CI: 1.16-1.68) with non-significant impacts on child's mental and physical health [31]. Programs offering additional social support for pregnant women at high risk for preterm or low birth weight (LBW) delivery showed significant impacts on reducing the likelihood of antenatal hospital admission (RR: 0.79, 95\% CI: 0.68-0.92) and cesarean birth (RR: 0.87, 95\% CI: 0.78-0.97) when compared to routine care [34]. Specialist out-reach clinics did not have any impact on health outcomes but improved compliance to treatment (RR: 0.63, 95\% CI: 0.52-0.77), patient-provider satisfaction (RR: 0.43, 95\% CI: 0.29-0.62) and access [33].

\section{Task shifting}

We included six [41,46-50] reviews pertaining to task shifting with a median score of 10 on AMSTAR criteria. Four reviews evaluating the impact of task shifting to $\mathrm{CHW}$ and midwives in $\mathrm{LMIC}$ reported $\mathrm{MNH}$ outcomes while the other two reviews from HIC focused on the impact of dietary counseling delivered through dietician versus nurses/doctors [50] and impact of nurses working as substitutes for primary care doctors [48]. Table 2 summarizes the characteristics of the included reviews.

Table 2 Characteristics of the included reviews for Human Resources-Task Shifting

\begin{tabular}{|c|c|c|c|c|c|c|c|}
\hline \multirow{2}{*}{$\begin{array}{c}\text { Reviews } \\
(n=06)\end{array}$} & \multirow{2}{*}{$\begin{array}{l}\text { Description of included } \\
\text { interventions }\end{array}$} & \multirow{2}{*}{$\begin{array}{l}\text { Type of } \\
\text { studies } \\
\text { included } \\
\text { (no) }\end{array}$} & \multirow{2}{*}{$\begin{array}{l}\text { Targeted health } \\
\text { care providers }\end{array}$} & \multicolumn{2}{|c|}{ Outcome reported } & \multirow{2}{*}{$\begin{array}{l}\text { Pooled } \\
\text { data } \\
(\mathrm{Y} / \mathrm{N})\end{array}$} & \multirow[t]{2}{*}{ Results } \\
\hline & & & & $\begin{array}{c}\text { Other } \\
\text { outcomes }\end{array}$ & $\begin{array}{l}\text { MNCH specific } \\
\text { outcomes }\end{array}$ & & \\
\hline \multirow[t]{8}{*}{$\begin{array}{c}\text { Bhutta } \\
2012[71]\end{array}$} & $\begin{array}{l}\text { Mid-level healthcare provider } \\
\text { defined as those who have } \\
\text { received less training than } \\
\text { doctors but who perform aspects } \\
\text { of doctors' tasks. }\end{array}$ & $\begin{array}{c}\text { RCT/CRCT: } \\
52 \\
\text { ITS: } 02 \\
\text { Case } \\
\text { Control:01 } \\
\text { Before } \\
\text { After: } 01\end{array}$ & $\begin{array}{l}\text { Nurse, midwives, } \\
\text { auxillary nurse, } \\
\text { auxillary nurse } \\
\text { midwife, surgical } \\
\text { technicians in both } \\
\text { HIC and LMIC }\end{array}$ & & $\begin{array}{l}\text { Wives versus } \\
\text { doctors + } \\
\text { midwives: }\end{array}$ & Yes & \\
\hline & & & & & $\begin{array}{c}\text { Rate of performing } \\
\text { c- section }\end{array}$ & & $0.92(0.81-1.15)$ \\
\hline & & & & & $\begin{array}{l}\text { Postpartum } \\
\text { hemorrhage }\end{array}$ & & $1.03(0.82-1.29)$ \\
\hline & & & & & $\begin{array}{l}\text { Overall fetal or } \\
\text { neonatal deaths }\end{array}$ & & $0.95(0.69-1.30)$ \\
\hline & & & & & Preterm births & & $0.87(0.73-1.04)$ \\
\hline & & & & & $\begin{array}{c}\text { Admission to } \\
\text { neonatal intensive } \\
\text { care }\end{array}$ & & $1.03(0.77-1.38)$ \\
\hline & & & & & $\begin{array}{c}\text { The use of } \\
\text { intrapartum regional } \\
\text { analgesia }\end{array}$ & & $0.88(0.81-0.96)$ \\
\hline & & & & & Episiotomies & & $0.83(0.77-0.90)$ \\
\hline
\end{tabular}




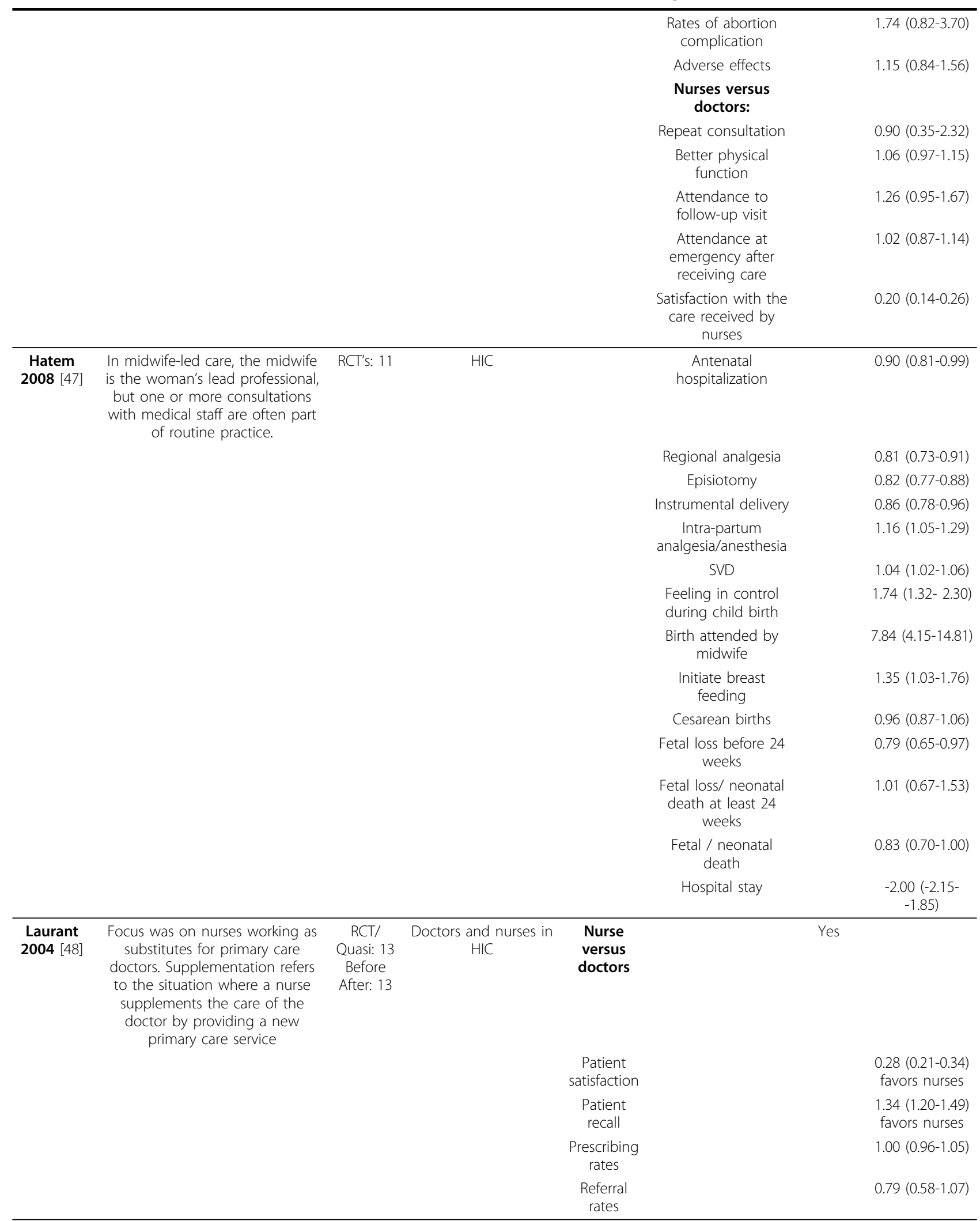


Table 2 Characteristics of the included reviews for Human Resources-Task Shifting (Continued)

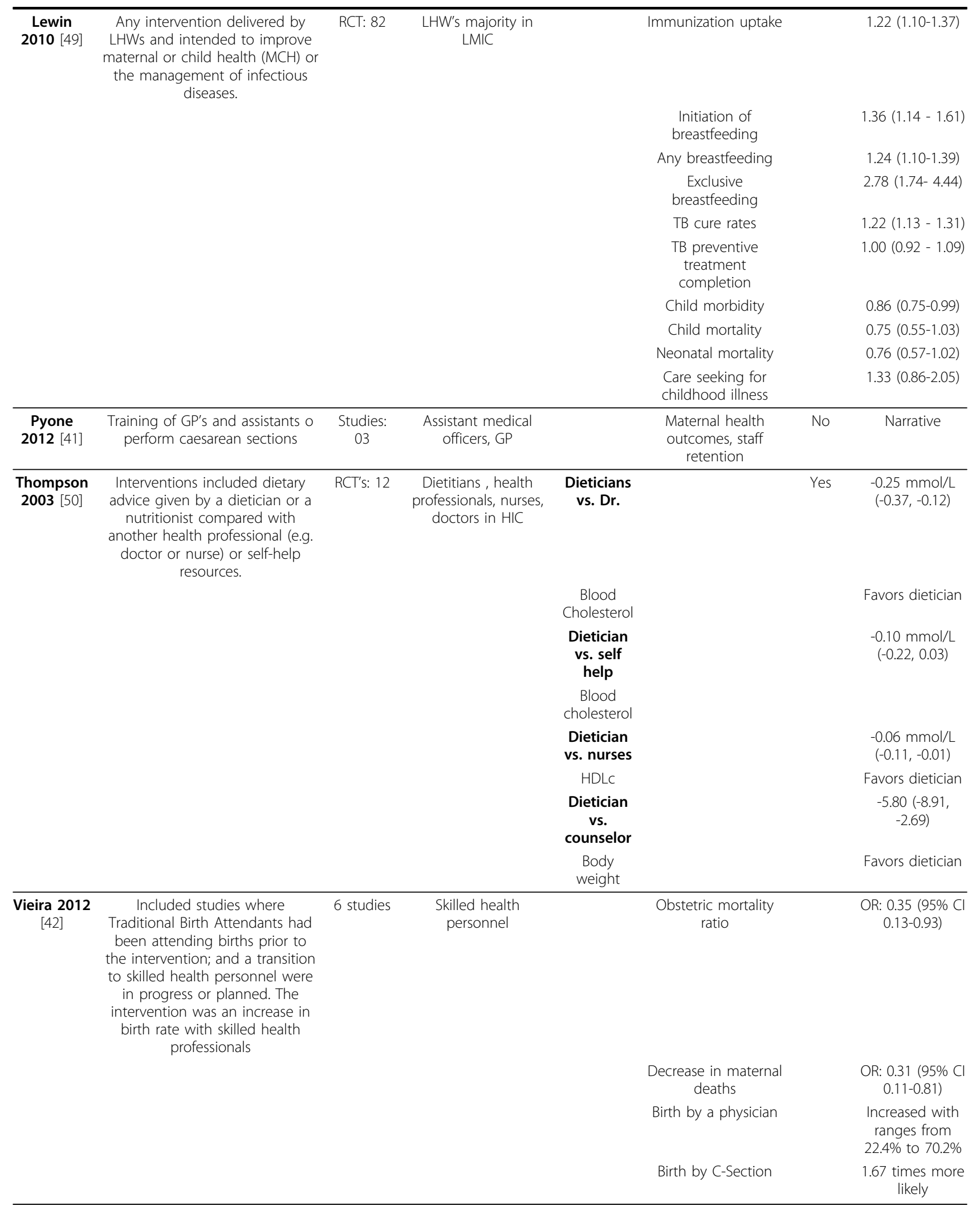


Care provided by midwives was found to be associated with significant improvements in antenatal hospitalization (RR: 0.90, 95\% CI: 0.81-0.99), episiotomy (RR: 0.81, 95\% CI: 0.77-0.88), instrumental delivery (RR: 0.86, 95\% CI: 0.78-0.96), initiation of breast feeding (RR: 1.35, 95\% CI: 1.03-1.76) and hospital stay (MD: $-2.00,95 \%$ CI: -2.15 to -1.85) [47]. Another review evaluating the effects of CHW interventions reported significant impacts on immunization uptake (RR: 1.22, 95\% CI: 1.10-1.37), breast feeding initiation (RR: $1.36,95 \% \mathrm{CI}: 1.14-1.61$ ), child morbidity (RR: 0.86, RR: 0.75-0.99) and TB cure rates (RR: 1.22 , 95\% CI: 1.13-1.31) compared to routine care [49]. Care delivered by MLHW versus non-MLHW was found comparable for antenatal hospitalization, antepartum hemorrhage, fetal loss/ neonatal deaths, induction of labour, spontaneous vaginal delivery, instrumental vaginal births, cesarean sections, perineal laceration requiring suturing, post-partum hemorrhage, preterm birth, LBW and admission to neonatal special/ intensive care unit. Furthermore, ANC provided by midwives alone gave comparable results on a range of $\mathrm{MNH}$ outcomes compared to care provided by doctors working in a team with midwives. These findings suggest that care delivered by MLHW can be safe and effective [46]. Improved patient satisfaction and recall was reported when nurses were substituted for primary health care provision in place of doctors; although the data was from HIC only [48]. In another review, dietary counseling given by dieticians was comparable to that by nurses or doctors [50].

\section{Training of human resources}

We found eighteen [23,39,51-66] reviews on human resource training with median quality score of 8.5 on AMSTAR rating scale. Three reviews reported $\mathrm{MNH}$ specific outcomes including ANC, institutional delivery, cesarean-section rates-section rates, referrals, stillbirths, maternal, perinatal and neonatal mortality while other reported outcomes included knowledge, compliance, performance and patient satisfaction. Most of the reviews evaluating training programs for outreach workers, $\mathrm{CHW}$, community midwives or TBA were conducted in LMIC while reviews on the training of other licensed healthcare professional like physicians, residents, fellows, and medical students were from HIC. Table 3 summarizes the characteristics of the included reviews.

In LMIC settings, training TBA (for providing basic antenatal, natal and postnatal care; preventive essential newborn care, breastfeeding counseling; management and referral of sick newborns; skills development in behavior change communication and community mobilization strategies to promote birth and newborn care preparedness) as a part of community based intervention packages showed significant improvement in referrals (RR: 1.4,

Table 3 Characteristics of the included reviews for human resources-training

\begin{tabular}{|c|c|c|c|c|c|c|c|}
\hline \multirow{2}{*}{$\begin{array}{c}\text { Reviews } \\
(n=18)\end{array}$} & \multirow{2}{*}{$\begin{array}{l}\text { Description of included } \\
\text { interventions }\end{array}$} & \multirow{2}{*}{$\begin{array}{l}\text { Type of } \\
\text { studies } \\
\text { included } \\
\text { (no) }\end{array}$} & \multirow{2}{*}{$\begin{array}{l}\text { Targeted health care } \\
\text { providers }\end{array}$} & \multicolumn{2}{|c|}{ Outcome reported } & \multirow{2}{*}{$\begin{array}{c}\text { Pooled } \\
\text { data } \\
(\mathrm{Y} / \mathrm{N})\end{array}$} & \multirow[t]{2}{*}{ Results } \\
\hline & & & & Other outcomes & $\begin{array}{c}\text { MNCH } \\
\text { specific } \\
\text { outcomes }\end{array}$ & & \\
\hline \multirow[t]{6}{*}{$\begin{array}{c}\text { Bhutta } 2010 \\
{[51]}\end{array}$} & $\begin{array}{l}\text { In-service training to health } \\
\text { personnel only, defined as SBAs } \\
\text { (nurses, midwives, doctors or } \\
\text { health personnel with midwifery } \\
\text { skills) for better maternal } \\
\text { outcomes. }\end{array}$ & $\begin{array}{c}\text { Before } \\
\text { after:08, } \\
\text { Quais:02, } \\
\text { Cross- } \\
\text { sectional: } 2\end{array}$ & $\begin{array}{c}\text { Skilled birth } \\
\text { attendants (doctors, } \\
\text { nurses and midwives) } \\
\text { as well as to other } \\
\text { service providers (lab } \\
\text { tech) in LMIC }\end{array}$ & & $\begin{array}{l}\text { Cesarean } \\
\text { section }\end{array}$ & No & $1.78(0.34-9.32)$ \\
\hline & & & & & $\begin{array}{l}\text { Maternal } \\
\text { mortality }\end{array}$ & & $0.57(0.36-0.91)$ \\
\hline & & & & & $\begin{array}{c}\text { Obstetric } \\
\text { complications }\end{array}$ & & $1.72(0.72-4.10)$ \\
\hline & & & & & $\begin{array}{l}\text { Institutional } \\
\text { delivery }\end{array}$ & & $2.92(2.09-4.06)$ \\
\hline & & & & & Referrals & & $0.57(0.25-1.31)$ \\
\hline & & & & & $\begin{array}{l}\text { Mean } \\
\text { antenatal } \\
\text { visits }\end{array}$ & & $0.90(0.47-1.33)$ \\
\hline $\begin{array}{l}\text { Giguere } \\
2012[72]\end{array}$ & $\begin{array}{l}\text { The distribution of published or } \\
\text { printed recommendations for } \\
\text { clinical care and evidence to } \\
\text { inform practice, including clinical } \\
\text { practice guidelines, journals and } \\
\text { monographs. }\end{array}$ & $\begin{array}{l}14 \text { RCTS } \\
31 \text { ITS }\end{array}$ & $\begin{array}{l}\text { All health care } \\
\text { professionals }\end{array}$ & $\begin{array}{l}\text { PEM vs. no } \\
\text { intervention }\end{array}$ & & Yes & \\
\hline
\end{tabular}


Table 3 Characteristics of the included reviews for human resources-training (Continued)

\begin{tabular}{|c|c|c|c|c|c|c|}
\hline & & & & $\begin{array}{c}\text { Practice } \\
\text { outcomes: } \\
\text { (categorical) }\end{array}$ & & $\begin{array}{c}\text { Median } \\
\text { absolute risk } \\
\text { difference } 0.02\end{array}$ \\
\hline & & & & $\begin{array}{l}\text { Profession } \\
\text { practice } \\
\text { outcomes: } \\
\text { (continuous) }\end{array}$ & & $\begin{array}{c}\text { median } \\
\text { improvement } \\
\text { in standardised } \\
\text { mean } \\
\text { difference } 0.13 \\
\text { (range }-0.16 \text {, } \\
0.36 \text { ) } \\
\end{array}$ \\
\hline $\begin{array}{l}\text { Forsetlund } \\
2009[53]\end{array}$ & $\begin{array}{l}\text { We included the following types } \\
\text { of educational meetings: } \\
\text { conferences, lectures, workshops, } \\
\text { seminars, symposia, and courses. }\end{array}$ & Trials: 81 & $\begin{array}{l}\text { Qualified health } \\
\text { professionals or health } \\
\text { professionals in } \\
\text { postgraduate training } \\
\text { mostly in HIC }\end{array}$ & $\begin{array}{l}\text { Any } \\
\text { intervention } \\
\text { with } \\
\text { educational } \\
\text { meeting vs. no } \\
\text { intervention: }\end{array}$ & Yes & $6 \%(1.8-15.9)$ \\
\hline & & & & Compliance & & \\
\hline & & & & $\begin{array}{l}\text { Only } \\
\text { educational } \\
\text { meeting vs. no } \\
\text { intervention: }\end{array}$ & & \\
\hline & & & & Compliance & & $6 \%(2.9-15.3)$ \\
\hline & & & & $\begin{array}{l}\text { Achievement of } \\
\text { treatment goal }\end{array}$ & & $3(0.1-4)$ \\
\hline $\begin{array}{l}\text { Hulscher } \\
2005[54]\end{array}$ & $\begin{array}{l}\text { Within the professional oriented } \\
\text { interventions we distinguished } \\
\text { between conceptually different } \\
\text { interventions: information } \\
\text { transfer, learning through social } \\
\text { influence, feedback and } \\
\text { reminders. }\end{array}$ & $\begin{array}{l}\text { RCT: } 37 \\
\text { Quasi: } 18\end{array}$ & $\begin{array}{c}\text { Family physicians, } \\
\text { general internists, } \\
\text { gynaecologists, } \\
\text { obstetricians, } \\
\text { pediatricians and } \\
\text { sometimesother } \\
\text { professionals like } \\
\text { nurse practitioners } \\
\text { and radiologists in HIC }\end{array}$ & $\begin{array}{l}\text { Preventive } \\
\text { services: }\end{array}$ & & \\
\hline & & & & $\begin{array}{l}\text { Group education } \\
\text { vs. no } \\
\text { intervention }\end{array}$ & & $\begin{array}{c}\text { Range: }-4 \% \text { - } \\
31 \%\end{array}$ \\
\hline & & & & $\begin{array}{l}\text { Multifaceted } \\
\text { interventions } \\
\text { versus group } \\
\text { education }\end{array}$ & & $\begin{array}{c}\text { Range: }-31 \% \text { - } \\
28 \%\end{array}$ \\
\hline $\begin{array}{c}\text { Hyde } 2000 \\
{[55]}\end{array}$ & $\begin{array}{c}\text { Critical appraisal is the process of } \\
\text { assessing and interpreting } \\
\text { evidence by systematically } \\
\text { considering its validity, results } \\
\text { and relevance to an individual's } \\
\text { work. }\end{array}$ & $\begin{array}{l}\text { RCT:01 } \\
\text { NRCT: } 08 \\
\text { CBA: } 07\end{array}$ & $\begin{array}{l}\text { Doctors, midwives, } \\
\text { managers and } \\
\text { researchers }\end{array}$ & Knowledge & Yes & $0.10(0.06-0.14)$ \\
\hline & & & & Skills & & $\begin{array}{l}14 / 16 \\
\text { comparisons } \\
\text { showed } \\
\text { positive effect }\end{array}$ \\
\hline & & & & Attitude & & $\begin{array}{c}4 / 4 \\
\text { comparisons } \\
\text { showed } \\
\text { positive } \\
\text { impact }\end{array}$ \\
\hline
\end{tabular}


Table 3 Characteristics of the included reviews for human resources-training (Continued)

\begin{tabular}{|c|c|c|c|c|c|c|c|}
\hline \multirow[t]{6}{*}{$\begin{array}{c}\text { Lassi } 2010 \\
{[39]}\end{array}$} & $\begin{array}{c}\text { Intervention packages that } \\
\text { included additional training of } \\
\text { outreach workers namely, female } \\
\text { health workers/visitors, } \\
\text { community midwives, } \\
\text { community/village health } \\
\text { workers, facilitators or TBAs in } \\
\text { maternal care during pregnancy, } \\
\text { delivery and in the postpartum } \\
\text { period; and routine newborn } \\
\text { care. }\end{array}$ & $\begin{array}{l}18 \text { cluster- } \\
\text { randomized/ } \\
\text { quasi- } \\
\text { randomized } \\
\text { trials }\end{array}$ & $\begin{array}{c}\text { Outreach workers } \\
\text { namely, female health } \\
\text { workers/visitors, } \\
\text { community midwives, } \\
\text { community/village } \\
\text { health workers, } \\
\text { facilitators or TBAs in } \\
\text { LMIC }\end{array}$ & & $\begin{array}{l}\text { Maternal } \\
\text { mortality }\end{array}$ & Yes & $0.77(0.59-1.02)$ \\
\hline & & & & & $\begin{array}{l}\text { Maternal } \\
\text { morbidity }\end{array}$ & & $0.75(0.61-0.92)$ \\
\hline & & & & & $\begin{array}{l}\text { Neonatal } \\
\text { mortality }\end{array}$ & & $0.76(0.68-0.84)$ \\
\hline & & & & & $\begin{array}{l}\text { Perinatal } \\
\text { mortality }\end{array}$ & & $0.80(0.71-0.91)$ \\
\hline & & & & & Referral & & $1.4(1.19-1.65)$ \\
\hline & & & & & $\begin{array}{l}\text { Early breast } \\
\text { feeding }\end{array}$ & & $1.94(1.56-2.42)$ \\
\hline \multirow[t]{3}{*}{$\begin{array}{c}\text { Légaré } 2010 \\
{[56]}\end{array}$} & $\begin{array}{l}\text { Interventions may include but } \\
\text { are not limited to the } \\
\text { distribution of printed } \\
\text { educational material, educational } \\
\text { meetings, audit and feedback, } \\
\text { reminders, and patient-mediated } \\
\text { interventions }\end{array}$ & RCT's:05 & $\begin{array}{c}\text { Healthcare } \\
\text { professionals, } \\
\text { residents, fellows, and } \\
\text { other pre } \\
\text { licensurehealthcare } \\
\text { professional }\end{array}$ & $\begin{array}{l}\text { Adoption of } \\
\text { shared decision } \\
\text { making: }\end{array}$ & & No & \\
\hline & & & & $\begin{array}{l}\text { Both patient } \\
\text { mediated } \\
\text { interventions }\end{array}$ & & & $1.06(0.62-1.5)$ \\
\hline & & & & $\begin{array}{l}\text { Multifaceted } \\
\text { intervention vs } \\
\text { usual care }\end{array}$ & & & $2.11(1.3-2.9)$ \\
\hline \multirow[t]{2}{*}{$\begin{array}{l}\text { Lugtenberg } \\
2008[57]\end{array}$} & $\begin{array}{c}\text { CPGs were defined as } \\
\text { "systematically developed } \\
\text { statements to assist practitioner } \\
\text { decisions about appropriate } \\
\text { healthcare for specific clinical } \\
\text { circumstances." }\end{array}$ & $\begin{array}{c}\text { CRCT: 10, } \\
\text { before after: } \\
\text { 10, ITS: } 1\end{array}$ & Physicians & $\begin{array}{l}\text { Process } \\
\text { outcomes }\end{array}$ & & No & $\begin{array}{l}\text { 17/19 studies } \\
\text { showed } \\
\text { significant } \\
\text { improvements }\end{array}$ \\
\hline & & & & $\begin{array}{l}\text { Patient } \\
\text { outcomes }\end{array}$ & & & $\begin{array}{c}6 / 9 \text { studies } \\
\text { showed } \\
\text { significant but } \\
\text { small } \\
\text { improvements }\end{array}$ \\
\hline \multirow[t]{2}{*}{$\begin{array}{l}\text { Norman } \\
1998[58]\end{array}$} & $\begin{array}{l}\text { The conscientious explicit and } \\
\text { judicious use of current evidence } \\
\text { in making decisions about the } \\
\text { care of individual patients }\end{array}$ & $\begin{array}{c}\text { RCT: } 03 \\
\text { CT:06 } \\
\text { Cohort: } 01\end{array}$ & $\begin{array}{l}\text { Medical students, } \\
\text { residents }\end{array}$ & $\begin{array}{l}\text { Undergraduate } \\
\text { knowledge }\end{array}$ & & No & $\begin{array}{c}\text { Mean gain } \\
\text { 17.0\%; [SD] } \\
4.0 \%)\end{array}$ \\
\hline & & & & $\begin{array}{c}\text { Residents } \\
\text { knowledge }\end{array}$ & & & $\begin{array}{c}\text { Mean gain } \\
\text { 1.3\%; SD 1.7\%) }\end{array}$ \\
\hline \multirow[t]{3}{*}{$\begin{array}{l}\text { O'Brien } \\
2007[59]\end{array}$} & $\begin{array}{l}\text { Educational outreach visits, } \\
\text { defined as use of a trained } \\
\text { person from outside the practice } \\
\text { setting who meets with } \\
\text { healthcare professionals in their } \\
\text { practice settings to provide } \\
\text { information with the intent of } \\
\text { changing their performance. }\end{array}$ & RCT: 69 & $\begin{array}{l}\text { Healthcare } \\
\text { professionals }\end{array}$ & Compliance & & Yes & $5.6 \%(3.0-9.0 \%)$ \\
\hline & & & & Prescribing & & & $4.8 \%(3.0-6.5 \%)$ \\
\hline & & & & $\begin{array}{l}\text { Professional } \\
\text { Performance }\end{array}$ & & & $6.0 \%(3.6-(16.0)$ \\
\hline
\end{tabular}


Table 3 Characteristics of the included reviews for human resources-training (Continued)

\begin{tabular}{|c|c|c|c|c|c|c|c|}
\hline \multirow[t]{2}{*}{$\begin{array}{l}\text { Opiyo } 2010 \\
{[60]}\end{array}$} & $\begin{array}{c}\text { Following in-service training } \\
\text { courses aimed at changing } \\
\text { provider behavior in the care of } \\
\text { the seriously ill newborn or child: } \\
\text { Neonatal and pediatric life } \\
\text { support courses e.g. NLS, NRP, } \\
\text { PALS, PLS, and others. Life } \\
\text { support elements. Other in- } \\
\text { service newborn and child } \\
\text { health training courses aimed at } \\
\text { the recognition and } \\
\text { management of the seriously ill } \\
\text { child }\end{array}$ & $\mathrm{RCT}: 02$ & $\begin{array}{l}\text { Doctors (general } \\
\text { practitioners and } \\
\text { specialists), nurses, } \\
\text { pharmacists and } \\
\text { dieticians/nutritionists, } \\
\text { in outpatient or } \\
\text { hospital-based } \\
\text { settings in LMIC }\end{array}$ & & $\begin{array}{l}\text { Performance } \\
\text { of adequate } \\
\text { initial } \\
\text { resuscitation } \\
\text { steps }\end{array}$ & No & $2.45(1.75-3.42)$ \\
\hline & & & & & $\begin{array}{c}\text { Frequency of } \\
\text { inappropriate } \\
\text { and } \\
\text { potentially } \\
\text { harmful } \\
\text { practices }\end{array}$ & & $0.40(0.13-0.66)$ \\
\hline $\begin{array}{l}\text { Oxman } \\
1995[61]\end{array}$ & $\begin{array}{l}\text { Participation of health care } \\
\text { providers in conferences, } \\
\text { lectures, workshops or } \\
\text { traineeships outside their } \\
\text { practice settings. }\end{array}$ & Trials: 17 & $\begin{array}{l}\text { General healthcare } \\
\text { providers }\end{array}$ & $\begin{array}{c}\text { Change in } \\
\text { health outcome } \\
\text { and } \\
\text { performance }\end{array}$ & & No & Narrative \\
\hline \multirow[t]{3}{*}{$\begin{array}{l}\text { Reeves } \\
2008[62]\end{array}$} & $\begin{array}{l}\text { An IPE intervention occurs when } \\
\text { members of more than one } \\
\text { health and/or social care } \\
\text { profession learn interactively } \\
\text { together, for the explicit purpose } \\
\text { of improving inter-professional } \\
\text { collaboration and/or the health/ } \\
\text { well being of patients/clients. }\end{array}$ & $\begin{array}{l}\text { RCT: } 04 \\
\text { CBA: } 02\end{array}$ & $\begin{array}{l}\text { Health and social care } \\
\text { professionals }\end{array}$ & $\begin{array}{c}\text { Patient } \\
\text { satisfaction }\end{array}$ & & No & $\begin{array}{l}4 / 6 \text { reported } \\
\text { positive } \\
\text { outcomes }\end{array}$ \\
\hline & & & & $\begin{array}{l}\text { Collaborative } \\
\text { team behavior }\end{array}$ & & & \\
\hline & & & & $\begin{array}{l}\text { Reduction in } \\
\text { clinical error }\end{array}$ & & & \\
\hline \multirow[t]{9}{*}{$\begin{array}{c}\text { Sibley } 2012 \\
{[73]}\end{array}$} & Trained birth attendants training & RCT: 6 & $\begin{array}{l}\text { Trained birth } \\
\text { attendants }\end{array}$ & & $\begin{array}{l}\text { Trained birth } \\
\text { attendants } \\
\text { versus } \\
\text { untrained } \\
\text { birth } \\
\text { attendants: }\end{array}$ & No & $\begin{array}{l}\text { Adjusted OR } \\
(95 \% \mathrm{Cl})\end{array}$ \\
\hline & & & & & Still births & & $\begin{array}{l}0.69(0.57 \text { to } \\
0.83)\end{array}$ \\
\hline & & & & & $\begin{array}{l}\text { Perinatal } \\
\text { death }\end{array}$ & & $\begin{array}{l}0.70(0.59 \text { to } \\
0.83)\end{array}$ \\
\hline & & & & & $\begin{array}{l}\text { Maternal } \\
\text { mortality }\end{array}$ & & $\begin{array}{l}0.74(0.45 \text { to } \\
1.22)\end{array}$ \\
\hline & & & & & Referral & & $\begin{array}{l}1.50(1.18 \text { to } \\
1.90)\end{array}$ \\
\hline & & & & & $\begin{array}{l}\text { Neonatal } \\
\text { deaths }\end{array}$ & & $\begin{array}{l}0.71(0.61 \text { to } \\
0.82)\end{array}$ \\
\hline & & & & & $\begin{array}{l}\text { Obstructed } \\
\text { labor }\end{array}$ & & $\begin{array}{l}1.26(1.03 \text { to } \\
1.54)\end{array}$ \\
\hline & & & & & Hemorrhage & & $\begin{array}{l}0.61(0.47 \text { to } \\
0.79)\end{array}$ \\
\hline & & & & & $\begin{array}{l}\text { Puerperal } \\
\text { Sepsis }\end{array}$ & & $\begin{array}{c}0.17(0.13 \text { to } \\
0.23)\end{array}$ \\
\hline \multirow[t]{3}{*}{$\begin{array}{l}\text { Smits } 2002 \\
{[63]}\end{array}$} & $\begin{array}{l}\text { Educational intervention was } \\
\text { problem based learning }\end{array}$ & RCT's: 06 & $\begin{array}{c}\text { Post graduate } \\
\text { continuing education } \\
\text { in HIC }\end{array}$ & $\begin{array}{l}\text { Participant's } \\
\text { knowledge, } \\
\text { performance, } \\
\text { satisfaction }\end{array}$ & & No & Narrative \\
\hline & & & & Patients health & & & \\
\hline & & & & Follow-up & & & \\
\hline
\end{tabular}


Table 3 Characteristics of the included reviews for human resources-training (Continued)

\begin{tabular}{|c|c|c|c|c|c|c|}
\hline $\begin{array}{c}\text { Thomas } \\
1999 \text { [64] }\end{array}$ & $\begin{array}{l}\text { Effect of clinical guideline on } \\
\text { behavior of nurses, midwives or } \\
\text { PAM's, on patient outcomes }\end{array}$ & $\begin{array}{l}\text { RCT: } 13 \\
\text { CBA: } 2 \\
\text { ITS: } 03\end{array}$ & $\begin{array}{l}\text { Nursing, midwifery, } \\
\text { health visiting, } \\
\text { podiatry, speech and } \\
\text { language therapy, } \\
\text { physiotherapy and } \\
\text { occupational therapy, } \\
\text { pharmacy and } \\
\text { radiography }\end{array}$ & $\begin{array}{c}\text { General } \\
\text { effectiveness }\end{array}$ & $\mathrm{No}$ & Narrative \\
\hline $\begin{array}{l}\text { Wensing } \\
1998[65]\end{array}$ & $\begin{array}{l}\text { Information transfer through } \\
\text { group education, reading } \\
\text { material and patient education }\end{array}$ & $\begin{array}{l}\text { RCT: } 39 \\
\text { CBA: } 22\end{array}$ & Physicians in $\mathrm{HIC}$ & $\begin{array}{l}\text { Effectiveness } \\
\text { against the } \\
\text { reported } \\
\text { outcome } \\
\text { measures }\end{array}$ & No & Narrative \\
\hline $\begin{array}{c}\text { Worral } 1997 \\
{[66]}\end{array}$ & $\begin{array}{c}\text { Interventions to improve medical } \\
\text { practice like dissemination } \\
\text { strategies such as conferences or } \\
\text { mailing }\end{array}$ & 13 trials & Physicians in $\mathrm{HIC}$ & $\begin{array}{c}\text { Conditions } \\
\text { studies }\end{array}$ & $\mathrm{No}$ & $\begin{array}{l}5 / 13 \text { studies } \\
\text { showed } \\
\text { statistically } \\
\text { significant } \\
\text { results }\end{array}$ \\
\hline
\end{tabular}

95\% CI: 1.19-1.65) and early breast feeding rates (RR: 1.94, 95\% CI: 1.56-2.42) with significant reductions in maternal morbidity (RR: 0.75, 95\% CI: 0.61-0.92), neonatal mortality (RR: 0.76 95\% CI: 0.68-0.84) and perinatal mortality (RR: 0.80, 95\% CI: 0.71-0.91) [39]. TBA training also reduced peri-neonatal mortality however there was insufficient data to provide the evidence base needed to establish training effectiveness [23]. In-service training courses specifically directed to improve the management of critically ill newborns showed significant improvements in performance of initial resuscitation (RR: 2.45, 95\% CI: $1.75-3.42$ ) and reduced the frequency of inappropriate and potentially harmful practices (RR: 0.40 , 95\% CI: 0.13-0.66) [60] while in-service trainings for skilled birth attendants (doctors, nurses and midwives) were found to be associated with significant impacts on maternal mortality (RR: $0.57,95 \%$ CI: $0.36-0.91$ ) and institutional delivery (RR: 2.92, 95\% CI: 2.09-4.06) [51]. The impacts on obstetric complication, ceasarean sections, ANC and referrals were non-significant.

For outcomes other than $\mathrm{MNH}$, educational outreach visits and meetings were associated with improved compliance (5.6\%, Range: $3-9 \%$ ), prescription (4.8\%, Range: $3-$ $6.5 \%$ ), professional practice (6\%, Range: $3.6-16 \%$ ), and some of the patient healthcare outcomes $[53,59]$. The evidence for continuing medical education, problem based learning and clinical practice guideline implementation remained inconclusive $[63,64,66]$. The impact of critical appraisal teaching on physicians' behavior was mixed with positive impacts on improving knowledge (MD: 0.10, 95\% CI: 0.06-0.14), skills, and attitude [55].

\section{Community mobilization}

We found two [39,67] reviews evaluating the impact of community mobilization strategies and formation of community support groups with median quality score of
8 on AMSTAR criteria. Both the reviews reported the impacts on MNH outcomes with one from HIC and the other from LMIC. Table 4 summarizes the characteristics of the included reviews.

Community based intervention packages involving family members through community support and advocacy groups and community mobilization along with additional training of outreach workers was reported as one of the most successful strategies showing significant impacts on maternal morbidity (RR: 0.75, 95\% CI: 0.610.92), neonatal mortality (RR: $0.7695 \% \mathrm{CI}: 0.68-0.84$ ), perinatal mortality (RR: 0.80, 95\% CI: 0.71-0.91), referral (RR: 1.4, 95\% CI: 1.19-1.65) and early breast feeding (RR: 1.94, 95\% CI: 1.56-2.42) [39]. Another review reported increased uptake of mammogram ranging from $5 \%$ to $15 \%$ with the formation of community groups [67].

\section{Discussion}

There is a greater body of existing evidence on the effectiveness of community based inputs for improving $\mathrm{MNH}$ outcomes in LMIC compared to district and facility level inputs (discussed in papers 3 and 4) [68,69]. At community level, home visitation, community mobilization, women's support groups and training of the $\mathrm{CHW}$ and TBA have shown maximum impact on a range of $\mathrm{MNH}$ outcomes. Community based generation of funds for transportation has also shown significant impacts in resource limited settings of India and sub-Saharan Africa. Interventions delivered through midwives, $\mathrm{CHW}$ and MLHW have not only demonstrated comparable outcomes when compared to routine non-MLHW care delivery but also reported better results for some of the outcomes. Specialized outreach clinics, continuing medical education, problem based learning, clinical practice guideline implementation and critical appraisal showed inconclusive and mixed results. 
Table 4 Characteristics of the included reviews for Community Mobilization and Support Groups

\begin{tabular}{|c|c|c|c|c|c|c|c|}
\hline \multirow[t]{2}{*}{ Reviews ( $n=02$ ) } & \multirow{2}{*}{$\begin{array}{l}\text { Description of included } \\
\text { interventions }\end{array}$} & \multirow{2}{*}{$\begin{array}{l}\text { Type of } \\
\text { studies } \\
\text { included } \\
\text { (no) }\end{array}$} & \multirow{2}{*}{$\begin{array}{l}\text { Targeted health care } \\
\text { providers }\end{array}$} & \multicolumn{2}{|c|}{ Outcome reported } & \multirow{2}{*}{$\begin{array}{l}\text { Pooled } \\
\text { data } \\
(\mathrm{Y} / \mathrm{N})\end{array}$} & \multirow[t]{2}{*}{ Results } \\
\hline & & & & $\begin{array}{l}\text { Other } \\
\text { outcomes }\end{array}$ & $\begin{array}{c}\text { MNCH } \\
\text { specific } \\
\text { outcomes }\end{array}$ & & \\
\hline Jepson 2000 [67] & $\begin{array}{l}\text { Formation of a committee } \\
\text { of community } \\
\text { representatives, promotion } \\
\text { of the screening service, } \\
\text { and implementation of an } \\
\text { appointment system by the } \\
\text { committee }\end{array}$ & RCT: 02 & $\begin{array}{c}\text { All people eligible to } \\
\text { participate in a screening } \\
\text { program as defined by the } \\
\text { entry criteria for that } \\
\text { program, included } \\
\text { population groups such as } \\
\text { pregnant women, } \\
\text { neonates, children and } \\
\text { adults in HIC }\end{array}$ & & $\begin{array}{l}\text { Mammogram } \\
\text { uptake }\end{array}$ & No & Range: 5\%-15\% \\
\hline \multirow[t]{6}{*}{ Lassi 2010 [39] } & $\begin{array}{l}\text { Intervention packages that } \\
\text { included additional training } \\
\text { of outreach workers namely, } \\
\text { female health workers/ } \\
\text { visitors, community } \\
\text { midwives, community/ } \\
\text { village health workers, } \\
\text { facilitators or TBAs in } \\
\text { maternal care during } \\
\text { pregnancy, delivery and in } \\
\text { the postpartum period; and } \\
\text { routine newborn care. }\end{array}$ & $\begin{array}{l}18 \text { cluster- } \\
\text { randomized/ } \\
\text { quasi- } \\
\text { randomized } \\
\text { trials }\end{array}$ & $\begin{array}{l}\text { outreach workers namely, } \\
\text { female health workers/ } \\
\text { visitors, community } \\
\text { midwives, community/ } \\
\text { village health workers, } \\
\text { facilitators or TBAs in LMIC }\end{array}$ & & $\begin{array}{l}\text { Maternal } \\
\text { mortality }\end{array}$ & Yes & $0.77(0.59-1.02)$ \\
\hline & & & & & $\begin{array}{l}\text { Maternal } \\
\text { morbidity }\end{array}$ & & $0.75(0.61-0.92)$ \\
\hline & & & & & $\begin{array}{l}\text { Neonatal } \\
\text { mortality }\end{array}$ & & $0.76(0.68-0.84)$ \\
\hline & & & & & $\begin{array}{l}\text { Perinatal } \\
\text { mortality }\end{array}$ & & $0.80(0.71-0.91)$ \\
\hline & & & & & Referral & & $1.4(1.19-1.65)$ \\
\hline & & & & & $\begin{array}{l}\text { Early breast } \\
\text { feeding }\end{array}$ & & $1.94(1.56-2.42)$ \\
\hline
\end{tabular}

Although the process pathways for the effectiveness of community delivered interventions are uncertain, they seem to influence community awareness, behavior change and practices, such as accessing skilled birth, use of clean delivery kits, breastfeeding and care seeking for maternal and newborn illnesses. Our overview findings greatly add to the global evidence base of intervention and delivery strategies that may improve $\mathrm{MNH}$ outcomes. It implies that within the community level inputs, three interventions have unparalleled significance: first, $\mathrm{CHW}$ who provide primary health care, can mobilize community members and impart knowledge; second, training of and linkages to TBA can provide basic prenatal and obstetric care, as well as referrals where skilled birth attendants are absent; third, community support groups, especially women's groups, can empower communities and help in problem solving and planning to improve opportunities for women's health, as well as care for mothers and newborns.

Countries in Asia and sub-Saharan Africa are facing critical shortages of healthcare workers despite of bearing
$25 \%$ of the world's diseases burden [20]. Reasons behind the migration of professional healthcare force to richer countries is suggested to be lack of incentive, poor working conditions and fewer opportunities for promotions [70]. There is also an existing pull from HIC to recruit health workers from LMIC. Given the shortage of care providers and functional health facilities, and the deeply entrenched practices, there is much interest in community-based interventions and strategies for care. Increasing the number of skilled health workers, training and educating them, providing them with incentives and improving the infrastructure is what needs to be done in all the LMIC to make progress towards achieving the Millennium Development Goals (MDG) 4 and 5.The findings from this overview testifies that increasing the availability and training of the skilled health workers including TBA and CHW for adequately recognizing and managing obstetric complications can significantly reduce maternal and neonatal morbidity and mortality especially in the resource limited settings of Asia and Africa where the highest maternal mortality burden 
exists with limited resources to mobilize. The challenge is to incentivize these programs and link them with formal health systems to increase retention. In many countries $\mathrm{CHW}$ are not linked to national health systems and are expected to work as volunteers which is a major drawback. Another existing challenge is the variation in prerequisites, recruitment, training, supervision and workload of various cadres of community workers including $\mathrm{CHW}$, TBA and midwives. There is a need to streamline their functioning and delegate the activities to achieve efficient implementation and maximum impact.

With the established effectiveness of task shifting and training of $\mathrm{CHW}$, future studies should focus on the factors affecting the sustainability and cost effectiveness of these interventions when scaled up [46]. There is a dearth of information on costs and equity aspects of community based programs as only a few studies have reported the actual costs incurred for saving lives or averting deaths with the use of these strategies. Researchers should now facilitate cost-effectiveness studies and consequent meta-analysis by collecting and reporting cost effectiveness data in a standardized format [39]. Further work is also needed on nurse- based care delivery models including length and frequency of contact, type of approach (e.g. individual or group, behavioral therapy or instructional techniques), level of training of practitioner, patient satisfaction and initial characteristics of patients to establish equivalence in care with the physician- based model and also for program replication [50]. Formal monitoring and evaluation of programs especially referral interventions are also necessary to develop better understanding of how referral interventions work. There is lack of data to establish effectiveness of mass media campaigns and community education as single strategies.

Outreach services may confer the most benefit to access and health outcomes in rural and underprivileged settings hence there is a need for good comparative studies in resource deprived settings rather than in HIC [33]. Among the outreach workers in the LMIC, the role of TBA is pivotal as they remain a major maternity care provider and their services expand from birth attendance to include newborn and post natal care like bathing and massage, domestic chores, and provision of care during postnatal period. Despite of that, TBA training remains controversial in relation to the global 'Safe Motherhood Initiative' as there is insufficient data to provide the evidence base needed to establish training effectiveness [23]. Hence methodologically rigorous evaluations with an adequate sample size are needed to measure the magnitude of the impact of TBA training on maternal and neonatal mortality.

Community based interventions have promising potential to provide range of services throughout the continuum of care and also reach the hard to reach population groups. Current evidence emphasizes that effective community based strategies exist to deliver a range of preventive and promotive interventions to improve the quality of care delivered for $\mathrm{MNH}$ and many of these interventions have the potential to reduce maternal, perinatal and neonatal morbidity and mortality. There is now a need to implement them on a larger scale throughout the LMIC. These interventions exist within the current health systems in most of the LMIC and hence policies are needed to integrate and sustain various task shifting and training interventions with the maternal health programs within their health systems. All stakeholders including governments, communities and donors need to work together to form these policies and develop models of health care to suit the needs of their own population. Still more work needs to be done in areas of recruitment, deployment and retention of the community based health care workers in the rural and underprivileged areas and improve the working conditions for them.

\section{Peer review}

Peer review reports are included in Additional file 1.

\section{Additional material}

Additional file 1:

List of abbreviations used

ANC: Antenatal Care; AMSTAR: Assessment of Multiple Systematic Reviews; CHW: Community Health Workers; Cl: Confidence Interval; ENC: Emergency Newborn Care; HIC: High Income Country; LBW: Low Birth Weight; LHV: Lady Health Visitor; LHW: Lay Health Workers; LMIC: Low and Middle Income Country; MD: Mean Difference; MLHW: Mid Level Healthcare Worker; MNH: Maternal Newborn Health; NLS: Newborn life Support; NRP: Neonatal Resuscitation Programs; PNC: Postnatal Care; RD: Risk Difference; RR: Relative Risk; SBA: Skilled Birth Attendant; TBA: Traditional Birth Attendant

\section{Competing interests}

We do not have any financial or non-financial competing interests for this review.

\section{Authors' contributions}

All authors contributed to the process and writing of the manuscript.

\section{Acknowledgments}

This work was supported by a grant from the Maternal health Task Force (MHTF) at the Harvard School of Public Health. We would like to acknowledge Rohail Kumar and Waleed Zahid who helped us in the search and abstraction of data.

\section{Declarations}

This article has been published as part of Reproductive Health Volume 11 Supplement 2, 2014: Quality of Care in Maternal and Child Health. The full contents of the supplement are available online at http://www.reproductivehealth-journal.com/supplements/11/S2.

\section{Authors' details}

'Division of Women \& Child Health, Aga Khan University, Karachi, Pakistan. ${ }^{2}$ Program for Global Pediatric Research, Hospital For Sick Children, Toronto. 
Published: 4 September 2014

\section{References}

1. Save the Children 2014: Ending newborn deaths: Ensuring every baby survives. 2014 [http://www.savethechildren.org.uk/resources/online-library/ ending-newborn-deaths], Access date: March 03, 2014.

2. UNICEF: Progress for children: achieving the MDGs with equity. New York: UNICEF; 2010 [http://www.unicef.org/protection/ Progress_for_Children-No.9_EN_081710.pdf], Access date: March 4, 2014

3. Ensor T, Cooper S: Overcoming barriers to health service access: influencing the demand side. Health Policy Plan 2004, 19:69-79.

4. Ray AM, Salihu HM: The impact of maternal mortality interventions using traditional birth attendants and village midwives. J Obstet Gynaecol 2004, 24(1):5-11.

5. Bhutta ZA, Das JK: Global burden of childhood diarrhea and pneumonia: what can and should be done? Pediatrics 2013, 131(4):634-636.

6. Bhutta ZA, Das JK, Rizvi A, Gaffey MF, Walker N, Horton S, Webb P, Lartey A, Black RE: Evidence-based interventions for improvement of maternal and child nutrition: what can be done and at what cost? Lancet 2013, 382(9890):452-477.

7. Bhutta ZA, Das JK, Walker N, Rizvi A, Campbell H, Rudan I, Black RE: Interventions to address deaths from childhood pneumonia and diarrhoea equitably: what works and at what cost? Lancet 2013, 381(9875):1417-1429.

8. Das JK, Lassi ZS, Salam RA, Bhutta ZA: Effect of community based interventions on childhood diarrhea and pneumonia: uptake of treatment modalities and impact on mortality. BMC Public Health 2013, 13(Suppl 3):S29.

9. Lehmann U, Dieleman M, Martineau T: Staffing remote rural areas in middle- and low-income countries: A literature review of attraction and retention. BMC Health Serv Res. 2008, 8(1):19.

10. Bhutta ZA, Das JK, Walker N, Rizvi A, Campbell H, Rudan I, Black RE: Interventions to address deaths from childhood pneumonia and diarrhoea equitably: what works and at what cost? Lancet 2013, 381(9875):1417-1429.

11. Chopra M, Munro S, Lavis JN, Vist G, Bennett S: Effects of policy options for human resources for health: an analysis of systematic reviews. Lancet 2008, 371:668-74.

12. WHO: Report of the WHO Task Force on Health Systems Research. 2005, Available at: http://www.who.int/rpc/ summit/en/Task Force on Health Systems Research.pdf.

13. WHO: The World Health Report 2006: working together for health. Geneva: World Health Organization 2006.

14. WHO: Task shifting: rational redistribution of tasks among health workforce teams. Geneva: World Health Organization 2007.

15. WHO/SEARO: Improving maternal, newborn and child health in the south-east Asia region - Bangladesh. [http://www.searo.who.int/LinkFiles/ Improving_maternal_newborn_and_child_health_bangladesh.pdf].

16. GHWA, WHO: Global Experience of Community Health Workers for Delivery of Health Related Millennium Development Goals: a Systematic Review, Country Case studies, and Recommendations for Scaling Up. 2010, Accessed from http://www.who.int/workforcealliance/knowledge/ publications/alliance/CHWreport_exsummary.pdf. Access date: March 6, 2014.

17. Lewin S, Munabi-Babigumira S, Glenton C, Daniels K, Bosch-Capblanch X, van Wyck BE, Odgaard-Jensen J, Johansen M, Aja GN, Zwarenstein M, Scheel IB: Lay health workers in primary and community health care for maternal and child health and the management of infectious diseases. Cochrane Database Syst Rev 2010, 3: CD004015, DOl: 10.1002/14651858. CD004015.pub3.

18. Bhutta ZA, Lassi ZS, Huicho L, Pariyo G: Global experience of community health workers for delivery of health related Millennium Development Goals: a systematic review, country case studies, and recommendations for integration into national health systems. Global Health Workforce Alliance; World Health Organization 2010.

19. Lehman U: Mid-level health workers-the state of the evidence on programmes, activities, costs and impact on health outcomes: a literature review. World Health Organization, Geneva 2008.

20. World Health Organization: A universal truth: no health without a workforce. 2013 [http://www.who.int/workforcealliance/knowledge/ resources/GHWA_AUniversalTruthReport.pdf.], Access date: March 5, 2014.
21. Kirigia JM, Gbary AR, Muthuri LK, Nyoni J, Seddoh A: The cost of health professionals' brain drain in Kenya. BMC Health Serv Res 2006, 6(1):89.

22. Ferrinho P, Van Lerberghe W, da Cruz Gomes A: Public and private practice: a balancing act for health staff. Bulletin of the World Health Organization 1999, 77(3):209.

23. Sibley LM, Sipe TA, Brown CM, Diallo MM, McNatt K, Harbarta N: Traditional birth attendant training for improving health behaviours and pregnancy outcomes. Cochrane Database Syst Rev 2007, 3(3):CD005460.

24. Lassi ZS, Majeed A, Rashid S, Yakoob MY, Bhutta ZA: The interconnections between maternal and newborn health-evidence and implications for policy. J Matern Fetal Neonatal Med 26(Suppl 1):3-53.

25. Rosato M, Laverack G, Grabman LH, Tripathy P, Nair N, Mwansambo C Azad K, Morrison J, Bhutta Z, Perry H, Rifkin S, Costello A: Alma-Ata: rebirth and revision 5. Community participation: lessons for maternal, newborn, and child health. Lancet 2008, 372:962-971.

26. Barros AJD, Ronsmans C, Axelson H, Loaiza E, Bertoldi AD, Fransa GVA, Bryce J, Boerma J, Victora CG: Equity in maternal, newborn, and child health interventions in Countdown to 2015: a retrospective review of survey data from 54 countries. Lancet 2012, 379(9822):1225-1233.

27. Austin A, Langer A, Salam RA, Lassi ZS, Das JK, Bhutta ZA, : Approaches to Improve Quality of Maternal and Newborn Health Care an overview of the evidence. An Overview of the Evidence 2014, 11(Suppl 1).

28. Shea BJ, Grimshaw JM, Wells GA, Boers M, Andersson N, Hamel C, Porter AC, Tugwell P, Moher D, Bouter LM: Development of AMSTAR: a measurement tool to assess the methodological quality of systematic reviews. BMC Med Res Methodol 2007, 7:10.

29. Blondel B, Breart $\mathrm{G}$ : Home visits during pregnancy: consequences on pregnancy outcome, use of health services, and women's situations. Semin Perinatol 1995, 19:263-271.

30. Ciliska D, Mastrilli P, Ploeg J, Hayward S, Brunton G, Underwood J: The effectiveness of home visiting as a delivery strategy for public health nursing interventions to clients in the prenatal and postnatal period: A systematic review. Prim Health Care Res Dev 2001, 2(1):41-54.

31. Elkan R, Kendrick D, Hewitt M, Robinson JJ, Tolley K, Blair M, Dewey M, Williams $D$, Brummell $K$ : The effectiveness of domiciliary health visiting: a systematic review of international studies and a selective review of the British literature. Health Technol Assess 2000, 4(13:i-v):41-54.

32. Gogia S, Sachdev HS: Home visits by community health workers to prevent neonatal deaths in developing countries: a systematic review. Bull World Health Organ 2010, 88(9):658-666.

33. Gruen RL, Weeramanthri TS, Knight SE, Bailie RS: Specialist outreach clinics in primary care and rural hospital settings. Cochrane Database Syst Rev 2003, 4(4):CD003798.

34. Hodnett ED, Fredericks S, Weston J: Support during pregnancy for women at increased risk of low birthweight babies. Cochrane Database Syst Rev 2010, 6(6):CD000198.

35. Hussein J, Kanguru L, Astin M, Munjanja S: The Effectiveness of Emergency Obstetric Referral Interventions in Developing Country Settings: A Systematic Review. PLoS Med 2012, 9(7):e1001264.

36. Hussein J, Kanguru L, Astin M, Munjanja S: What kinds of policy and programme interventions contribute to reductions in maternal mortality? Technical report London: EPPI-Centre, Social Science Research Unit: Institute of Education, University of London; 2011.; 2011.

37. Issel LM, Forrestal SG, Slaughter J, Wiencrot A, Handler A: A Review of Prenatal Homeâ€ Visiting Effectiveness for Improving Birth Outcomes. $J$ Obstet Gynecol Neonatal Nurs 2011, 40(2):157-165.

38. Kendrick D, Hewitt M, Dewey M, Elkan R, Blair M, Robinson J, Williams D, Brummell $K$ : The effect of home visiting programmes on uptake of childhood immunization: a systematic review and meta-analysis. J Public Health 2000, 22(1):90-98.

39. Lassi ZS, Haider BA, Bhutta ZA: Community-based intervention packages for reducing maternal and neonatal morbidity and mortality and improving neonatal outcomes. Cochrane Database Syst Rev 2010, 11(11):CD007754.

40. McNaughton DB: Nurse home visits to maternal-child clients: A review of intervention research. Public Health Nurs 2004, 21(3):207-219.

41. Pyone T, Sorensen BL, Tellier S: Childbirth attendance strategies and their impact on maternal mortality and morbidity in low-income settings: a systematic review. Acta Obstet Gynecol Scand 2012, 91(9):1029-1037.

42. Vieira C, Portela A, Miller T, Coast E, Leone T, Marston C: Increasing the use of skilled health personnel where traditional birth attendants were 
providers of childbirth care: a systematic review. PLoS One 2012, 7(10): e47946.

43. Peacock S, Konrad S, Watson E, Nickel D, Mahajarine N: Effectiveness of home visiting programs on child outcomes: a systematic review. BMC Public Health 2013, 13:17.

44. van Lonkhuijzen L, Stekelenburg J, van Roosmalen J: Maternity waiting facilities for improving maternal and neonatal outcome in low-resource countries. Cochrane Database Syst Rev 2012, 10(3).

45. Bull J, McCormick G, Swann C, Mulvihill C: Ante-and post-natal homevisiting programmes: a review of reviews [Evidence briefing]. London: Health Development Agency 2004.

46. Bhutta ZA, Lassi ZS: Global Experience of Mid-level Health Workers for Delivery of Health related Millennium Development Goals: A Systematic Review. Unpublished 2012.

47. Hatem M, Sandall J, Devane D, Soltani H, Gates S: Midwife-led versus other models of care for childbearing women (Review). Cochrane Database Syst Rev 2008, 4(4):CD004667.

48. Laurant M, Reeves D, Hermens R, Braspenning J, Grol R, Sibbald B: Substitution of doctors by nurses in primary care. Cochrane Database Syst Rev 2004, 4(4):CD001271.

49. Lewin S, Munabi-Babigumira S, Glenton C, Daniels K, Bosch-Capblanch X, van Wyck BE, Odgaard-Jensen J, Johansen M, Aja GN, Zwarenstein M, Scheel IB: Lay health workers in primary and community health care for maternal and child health and the management of infectious diseases. Cochrane Database Syst Rev 2010, 3: CD004015, DOI: 10.1002/14651858. CD004015.pub3.

50. Thompson RL, Summerbell CD, Hooper L, Higgins JP, Little PS, Talbot D, Ebrahim S: Dietary advice given by a dietitian versus other health professional or self-help resources to reduce blood cholesterol. Cochrane Database Syst Rev 2003, 3(3):CD001366.

51. Bhutta ZA, Lassi ZS, Mansoor N: Systematic Review on Human Resources for Health Interventions to Improve Maternal Health Outcomes: Evidence from Developing Countries. World Health Organization (WHO) 2010.

52. Farmer AP, Legare F, Turcot L, Grimshaw J, Harvey E, McGowan JL, Wolf F: Printed educational materials: effects on professional practice and health care outcomes. Cochrane Database Syst Rev 2009, 3(3):CD004398.

53. Forsetlund $L$, Bjorndal A, Rashidian A, Jamtvedt G, O'Brien MA, , Wolf $F$, Davis D, Odgaard-Jensen J, Oxman AD: Continuing education meetings and workshops: effects on professional practice and health care outcomes (Review). Cochrane Database Syst Rev 2009, 2(2):CD003030.

54. Hulscher M, Wensing M, Weijden T, Grol R: Interventions to implement prevention in primary care. Cochrane Database Syst Rev 2005, 1(1):CD000362.

55. Hyde C, Parkes J, Deeks J, Milne R: Systematic review of effectiveness of teaching critical appraisal. ICRF/NHS 2000.

56. Legare F, Ratte S, Stacey D, Kryworuchko J, Gravel K, Graham ID, Turcotte S: Interventions for improving the adoption of shared decision making by healthcare professionals. Cochrane Database Syst Rev 2010, 5(5):CD006732.

57. Lugtenberg M, Burgers JS, Westert GP: Effects of evidence-based clinical practice guidelines on quality of care: a systematic review. Quality and Safety in Health Care 2009, 18(5):385-392.

58. Norman GR, Shannon SI: Effectiveness of instruction in critical appraisal (evidence-based medicine) skills: a critical appraisal. CMAJ 1998, 158(2):177-181.

59. O'Brien MA, Rogers $\mathrm{S}$, Jamtvedt G, Oxman AD, Odgaard-Jensen J, Kristoffersen DT, Forsetlund L, Bainbridge D, Freemantle N, David DA: Educational outreach visits: effects on professional practice and health care outcomes. Cochrane Database Syst Rev 2007, 4(4):CD000409.

60. Opiyo N, English M: In-service training for health professionals to improve care of the seriously ill newborn or child in low and middleincome countries. Cochrane Database Syst Rev 2010, 4(4):CD007071.

61. Oxman AD, Thomson MA, Davis DA, Haynes RB: No magic bullets: a systematic review of 102 trials of interventions to improve professional practice. CMAJ 1995, 153(10):1423-1431.

62. Reeves S, Zwarenstien M, Goldman J, Barr H, Freeth D, Hammick M, Koppel I: Interprofessional education: effects on professional practice and health care outcomes. Cochrane Database Syst Rev 2008, 1(1):CD002213.

63. Smits PBA, Verbeek J, De Buisonje CD: Problem based learning in continuing medical education: a review of controlled evaluation studies. BMJ 2002, 324(7330):153-156
64. Thomas LH, McColl E, Cullum N, Rousseau N, Soutter J: Clinical guidelines in nursing, midwifery and the therapies: a systematic review. Journal of Advanced Nursing 1999, 30(1):40-50.

65. Wensing M, van der Weijden T, Grol R: Implementing guidelines and innovations in general practice: which interventions are effective? $\mathrm{Br} J$ Gen Pract 1998, 48(427):991-997.

66. Worrall G, Chaulk P, Freake D: The effects of clinical practice guidelines on patient outcomes in primary care: a systematic review. CMAJ 1997, 156(12):1705-1712

67. Jepson $\mathrm{R}$, et al: The determinants of screening uptake and interventions for increasing uptake: a systematic review. Health Technol Assess 2000, 4(14):1-133.

68. Salam RA, Lassi ZS, Das JK, Bhutta ZA: Evidence from district level inputs to improve quality of maternal and newborn health: Interventions and Findings. Reproductive Health 2014, 11(Suppl 3).

69. Das JK: Evidence from facility level inputs to improve quality of maternal and newborn health: Interventions and Findings. Reproductive Health 2014, 11(Suppl 4).

70. Falconer A, Crisp E, Warick C, Day-Strik F: Scaling up human resources for women's health. BJOG 2009, 116(Suppl 1):11-14

71. Bhutta ZA, Lassi ZS, Pariyo G, Huicho L: Global Experience of Community Health Workers for Delivery of Health Related Millennium Development Goals: A Systematic Review, Country Case Studies, and Recommendations for Integration into National Health Systems. Global Health Workforce Alliance, World Health Organization 2012

72. Giguere A, Legare F, Grimshaw J, Turcotte S, Fiander M, Grudniewicz A, Makosso-Kallyth S, Wolf FM, Farmer AP, Gagnon MP: Printed educational materials: effects on professional practice and healthcare outcomes. Cochrane Database Syst Rev 2012, 10:CD004398.

73. Sibley LM, Sipe TA, Brown CM, Diallo MM, McNatt K, Habarta N: Traditional birth attendant training for improving health behaviours and pregnancy outcomes. Cochrane Database Syst Rev 2007, 3.

doi:10.1186/1742-4755-11-S2-S2

Cite this article as: Lassi et al:: Evidence from community level inputs to improve quality of care for maternal and newborn health: interventions and findings. Reproductive Health 2014 11(Suppl 2):S2.

\section{Submit your next manuscript to BioMed Central and take full advantage of:}

- Convenient online submission

- Thorough peer review

- No space constraints or color figure charges

- Immediate publication on acceptance

- Inclusion in PubMed, CAS, Scopus and Google Scholar

- Research which is freely available for redistribution

Submit your manuscript at www.biomedcentral.com/submit
C Biomed Central 